\title{
Residential Services for Youth Impacted by Family Violence and Trauma: Implications for Child Welfare Policy and Practices
}

\author{
Jan Nisenbaum
}

Published online: 18 September 2013

(C) Springer Science+Business Media New York 2013

This issue of the Journal of Family Violence has addressed critical clinical, organizational and public policy issues in the treatment of traumatized children involved in the child welfare system. Each article provides important insight into the treatment needs of children and youth who have experienced trauma and provides enlightened guidance on creating beneficial environments to promote their health and well-being. The articles make a significant contribution to addressing the challenges inherent to the design and implementation of effective treatment modalities for children and youth requiring treatment in residential programs. The Massachusetts child welfare system has benefitted greatly from the expertise and dedication of the contributing authors in addressing the needs of youth who have experienced complex trauma.

Children and youth involved in the public child welfare system have frequently experienced a variety of traumatic events often resulting from chronic abuse or neglect. These youth present significant behavioral and treatment challenges to public school systems, the courts, as well as mental health and child welfare providers. Public and private agencies have consistently sought to address their multi-varied and complex treatment needs through a variety of residential and communitybased settings, utilizing a myriad of clinical approaches. Over the past decade, advances in our knowledge about the impact of trauma on neurological, emotional and behavioral development have led to substantially improved interventions and treatment modalities.

The Massachusetts Department of Children and Families (DCF) has undertaken several key initiatives over the past 4 years to build system capacity to provide trauma-informed child welfare services and to work collaboratively with mental

J. Nisenbaum $(\bowtie)$

Clinical and Program Services, Department of Children and Families, Rensselaer, NY, USA

e-mail: Jan.Nisenbaum@state.ma.us health agencies to provide trauma-specific treatment for children and families in their care or custody. In 2009, the Department implemented a new Integrated Casework Practice Model. The cornerstones of this new approach to child welfare casework practice included: 1) Positive Engagement and Empowerment of families, 2) Progressive Understanding of children and families strengths and needs, 3) Capacity Building to enhance parental capacities, and 4) Consolidating and Sustaining Gains prior to case closing. The clinical approaches Massachusetts has incorporated into the new casework practice model included safety-organized, trauma informed and solution focused strategies for enhancing our work with children and families. Through intentional training and coaching efforts, the Department has implemented an important change in our casework practice and the culture of the agency. As a result, the Department has improved outcomes in safety, permanency and well-being of children and families involved in the child welfare system.

The implementation of the new casework practice model was accompanied by an effort to align services within the child welfare provider network to deepen the understanding of the impact of trauma on children and families, and to focus interventions on treatment approaches that are consistent with emerging knowledge in this field. To support this effort, the Department of Children and Families, in collaboration with the Massachusetts Department of Mental Health, is in the process of implementing Caring Together, an innovative approach to the procurement and delivery of residential treatment services for children and youth served by both public agencies. One of the foundational principles in the design of the services offered through Caring Together has been the implementation of approaches that are trauma-informed and incorporate evidence-informed treatments and interventions.

These efforts have been greatly enhanced by the work of the Massachusetts Child Trauma Project. This unique public private partnership between the Department of Children and 
Families; LUK, Inc.; Justice Resource Institute Trauma Center; Boston Medical Center's Child Witness to Violence Program; and University of Massachusetts Medical School has been supported by a grant from the Administration for Children and Families: Children's Bureau. Massachusetts was one of five states to receive this grant to help build capacity within the child welfare system and mental health provider agencies to provide trauma-informed approaches and trauma-specific, evidence-based treatments. The grant is in the third year of implementation and has provided support for training of the child welfare staff, teams of clinicians from mental health provider agencies, and foster parents across the state.

The future success of child welfare systems in delivering services that result in positive outcomes for children, youth and families is dependent upon consistently enhancing our knowledge of the impact of traumatic experiences and ensuring that those services effectively address the resulting emotional and behavioral needs. These efforts are successful when there is dedicated collaboration between public and private sectors to assure that treatment interventions are aligned and that children and families experience consistent clinical approaches across the services and supports they receive. The commitment of public and private child welfare and mental health agencies to effectively addressing the traumatic experiences of children, youth and families involved in their respective systems is critical. Through collaborative and diligent attention to consistently aligning and improving our services and treatment interventions, the health and well-being of the children and families we serve will most certainly be improved. 